\title{
Terminología Morfológica Internacional: Algo Más que Anatomía, Histología y Embriología
}

\author{
International Morphological Terminology: More than Anatomy, Histology and Embryology
}

\author{
Ricardo Jorge Losardo, ${ }^{*, *, * * * * * * * *}$; Nadir Eunice Valverde Barbato de Prates ${ }^{*, * * *, * * * * * * * * * *}$; Manuel Arteaga-Martínez ${ }^{*, * * * *, * * * * * * * * * * * *}$; \\ Richard Haltí Cabral ${ }^{*, * * * * * * * * * * * * * *}$ \& María Isabel García-Peláez ${ }^{*, * * * * *, * * * * * * * * * *}$
}

LOSARDO, R. J.; PRATES, N. E. V. B.; ARTEAGA-MARTÍNEZ, M.; CABRAL, R. H. \& GARCÍA-PELÁEZ, M. I. Terminología morfológica internacional: algo más que anatomía, histología y embriología. Int. J. Morphol., 33(1):400-407, 2015.

RESUMEN: El estudio de la terminología morfológica internacional y su implementación en una normativa es complejo. La tarea que realiza el grupo de expertos morfólogos no es suficiente. Se explican algunos aspectos que nos acercarán al entendimiento de esta problemática y se muestra el camino para superar este desafío.

PALABRAS CLAVE: Terminología; Anatomía; Histología; Embriología; Morfología; Educación médica; Lingüística.

\section{INTRODUCCIÓN}

En los Simposios Ibero-Latinoamericanos de Terminología (SILAT), creados en el 2009 por la Asociación Panamericana de Anatomía (APA), se estudian los términos médicos utilizados en el campo de las ciencias morfológicas: anatomía, histología y embriología.

La comunidad científica de los países de habla hispana y portuguesa debe enfrentarse al problema que originan los diferentes términos que recibe una misma estructura. Ello se debe, por un lado, a la fragmentación de nuestras ciencias médicas y biológicas y, por el otro, al compartir un ámbito geográfico extenso como es la región ibero-latinoamericana.

El objetivo del SILAT es unificar, en esta región, las denominaciones de las estructuras morfológicas, que a su vez son la base de las ciencias médicas, con el fin de lograr un lenguaje científico común y facilitar el aprendizaje y la comunicación entre profesionales. Además, estudia, analiza, traduce, divulga y fomenta la terminología morfológica internacional de la Federación Internacional de Asociaciones de Anatomistas (IFAA).

Sin embargo, estas tareas de la APA (creada en 1966) y de la IFAA (creada en 1903) no terminan allí (Losardo, 2009; International Federation of Association of Anatomists, 1992). La terminología morfológica internacional luego debe trasladarse a los programas de enseñanza universitaria y a las instituciones de salud de todo el mundo, para que se aplique y de esta forma lograr su uso cotidiano (Losardo et al., 2010).

Esta última meta es más necesaria en nuestro ámbito ibero-latinoamericano. Para ello se necesita el trabajo conjunto del SILAT, de las sociedades científicas, de las universidades y de los ministerios de educación y salud de cada país para lograr que estos resultados tengan el impacto deseado. De esta manera, se reflejarán en el campo de la investigación, de la docencia y de la práctica médica asistencial, y así se facilitará el intercambio y el avance científico en Ibero-Latinoamérica.

Miembro del Consejo directivo de SILAT

** Presidente honorario de la Asociación Panamericana de Anatomía, Buenos Aires, Argetina.

**** $\quad$ Presidente SILAT IV, São Paulo, Brazil.

***** Presidente de SILAT VI y Presidente de la Asociación Panamericana de Anatomía, Mexico D.F., Mexico.

***** $\quad$ Presidente SILAT VII y Secretario General de la Asociación Panamericana de Anatomía, São Paulo, Brazil.

******* Presidente de SILAT IX y Vicepresidente de la Asociación Panamericana de Anatomía, Mexico D.F., Mexico.

******** Facultad de Medicina, Universidad del Salvador (USAL), Buenos Aires, Argentina.

********** Instituto de Ciências Biomédicas (ICB), Universidade de São Paulo (USP), São Paulo, Brasil.

Facultad de Medicina, Universidad Nacional Autónoma de México (UNAM), Mexico D.F., Mexico. 
Aunque hace años que se trabaja tanto a nivel regional como internacional para unificar los términos de estas estructuras, en la práctica aún no se ha conseguido un resultado totalmente satisfactorio (Duque Parra et al., 2006; Jiménez-Gutiérrez, 2009; Pawlina \& Drake, 2009; Vogl, 2009; Andriani, 2010; Cruz et al., 2010a; Algieri et al., 2013; Duque Parra \& Ríos, 2013; Vandaele \& Gingras Harvey, 2013; del Sol \& Vásquez, 2014; Vásquez \& del Sol, 2014).

Tal como se viene haciendo, el estudio de la terminología morfológica internacional y su implementación en una normativa es complejo. Se escapa a la tarea que realizan estos grupos de expertos morfólogos y también a aquellos profesionales de la salud que fomentan el uso de esa terminología.

En este trabajo se explican algunos aspectos que nos acercarán al entendimiento de este problema y cómo creemos que se puede superar.

\section{SITUACIÓN ACTUAL}

Estructura y funcionamiento . El FCAT, FICAT o FIPAT (denominaciones sucesivas de un mismo organismo), que depende de la Federación Internacional de Asociaciones de Anatomistas (IFAA) y que sucedió al International Anatomical Nomenclature Committee (IANC), está integrado por reconocidos profesores e investigadores que se reúnen periódicamente en distintos países para tratar la terminología morfológica. Como resultado de estas reuniones surgieron tres terminologías internacionales: la Anatómica, la Histológica y la Embriológica, reunidas cada una en un libro (FCAT, 1998; FICAT, 2008; FIPAT, 2011; FIPAT, 2013).

El SILAT tomó su modelo de las reuniones de los expertos de la FICAT y lo adaptó a la región. Funciona como un programa científico-educativo creado y supervisado por la Asociación Panamericana de Anatomía (Cruz et al., 2010a; Cruz et al., 2010b; Losardo et al.). Se inició en febrero del 2009 en San José de Costa Rica y su mentor fue un miembro de FCAT del área latinoamericana que en ese momento era presidente de la APA, el doctor Rolando Cruz Gutiérrez (Fig. 1).

Su funcionamiento se basa en la participación de profesores e investigadores universitarios de las ciencias morfológicas comprometidos en la temática, que se reúnen durante varias jornadas de trabajo. En ellas estudian y discuten los problemas terminológicos regionales e internacionales para lograr, en un marco participativo, la construcción colectiva de la terminología morfológica.
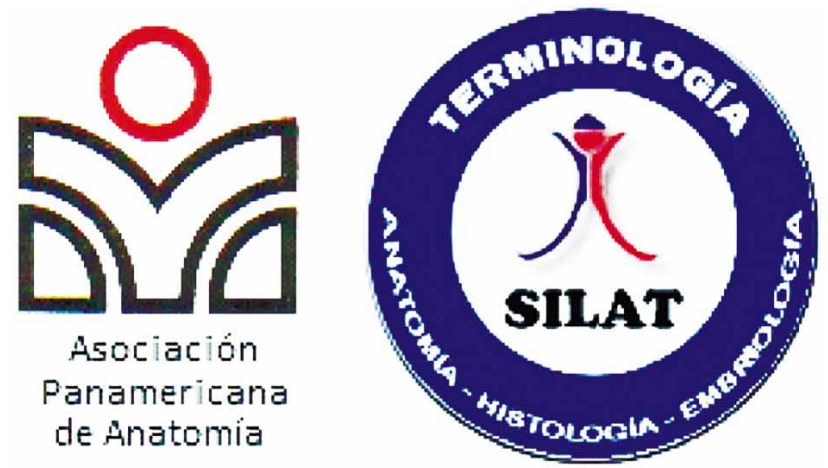

Fig.1. Logos de APA y SILAT.

Se realizan reuniones ordinarias con sedes en los distintos países participantes: Argentina, Bolivia, Brasil, Colombia, Costa Rica, Cuba, Chile, Ecuador, Estados Unidos de Norteamérica, El Salvador, Guatemala, Honduras, México, Nicaragua, Perú y Venezuela.

Cada simposio tiene su Presidente, que es nombrado por los países asistentes en la última reunión previa al evento y es el principal encargado de organizarlo (Cruz et al., 2010a; Losardo et al.).

A la fecha, se han llevado a cabo diez SILAT, dos en conjunto con el Congreso Panamericano de Anatomía, máximo encuentro científico de la Asociación Panamericana de Anatomía (2010, Chile y 2013, México) y otro con el International Symposium on Morphological Sciences (2012, Brasil), lo que demuestra su repercusión y reconocimiento internacional. También y a nivel más local, se realizó en forma conjunta con los Congresos de Anatomía del Cono Sur.

El SILAT tiene la particularidad de contar con dos representantes de la antigua FCAT, uno hispanoparlante y el otro lusoparlante. Ambos fueron presidentes de la APA. Ellos son los doctores Rolando Cruz Gutiérrez, de Costa Rica y José Carlos Prates, de Brasil. De esta manera, en su doble condición, conocen las problemáticas regionales e internacionales de la terminología morfológica.

\section{Acciones y objetivos}

Al momento de discutir la terminología morfológica internacional, la IFAA estableció que:

1. Casi todas las estructuras morfológicas se denominen con una sola palabra.

2. El lenguaje empleado sea el latín. 
3. Se describa el elemento de la forma más fiel a los principios de la geometría y la forma. El término debe concentrar la información y descripción de la estructura.

\section{Se eliminen las eponimias.}

5. El término sea congruente, es decir, que si se aceptan sus derivados se acepte la palabra primitiva que dio origen a aquellos derivados.

En cuanto a la denominación de las estructuras morfológicas, se respetan las raíces latinas, griegas y árabes y también se consideran las traducciones aceptadas por las reglas de la lingüística de los idiomas oficiales (Jiménez-Gutiérrez; Cruz et al., 2010a; Salgado \& Trujillo, 2010; Mejías, 2012).

Cada término es tratado como uniforme y estático en el tiempo, espacio y grupo social. Se eliminan los matices culturales regionales y las connotaciones afectivo-sociales. El término queda así "limpio".

Todos aquellos términos vernáculos y folklóricos y aquellos que llevan el nombre del anatomista que los descubrió e investigó se dejan de lado. Sin embargo, es importante preservarlos por lo que se propone volcarlos en otras publicaciones que tengan enfoque histórico: en diccionarios de regionalismos y en diccionarios de epónimos (Cruz et al., 2010a). SILAT principalmente vincula las listas latinas e inglesas con los idiomas español y portugués.

Además, las presentaciones hechas por los profesores e investigadores en los simposios sirven para conocer términos "panamericanos" surgidos en los laboratorios de disección y en los quirófanos, que no fueron contemplados en la edición española en circulación, traducción de la Terminología Anatómica Internacional (Sociedad Anatómica Española, 2001; Cruz et al., 2010a). El SILAT contribuye a enriquecer las futuras ediciones y darle una autoría más participativa y democrática.

Finalmente, cuando surgen diferencias técnicas que el Comité de Expertos de SILAT no puede resolver, son derivadas y planteadas a la FIPAT de la Federación Internacional de Asociaciones de Anatomistas (IFAA) contribuyendo con sugerencias y propuestas.

Más allá del encuentro de expertos. La función de los expertos en terminología es de alguna manera lograr un modelo idealizado, lo que debería ser y que perdure. Este objetivo no es fácil. El SILAT, siendo una entidad joven, advierte la problemática y en sus estatutos enuncia actividades que se extienden más allá del mismo simposio o encuentro (Cruz et al., 2010b; Losardo et al.), que son:
1. Promoción: coordinar y promover la difusión y utilización de la terminología internacional en las universidades.

2. Docencia: capacitar docentes en esta materia y formar instructores con el fin de lograr un efecto multiplicador.

3. Investigación: son estudios que sirven de base para la búsqueda de un lenguaje científico común para toda la comunidad científica ibero-latinoamericana. Además, se formulan propuestas de terminologías regionales y nacionales, que se discuten en el Comité de Expertos de SILAT.

4. Publicaciones: llevar a cabo una labor de documentación en el campo científico y educativo a través de publicaciones: informes de simposios, seminarios, jornadas, conferencias, mesas redondas, folletos, boletines, cuadernillos y libros sobre terminología.

5. Asesoría técnica: considerar y ofrecer en las universidades proyectos de asistencia y cooperación técnica en la materia.

En definitiva, lo que se persigue con estas actividades del SILAT es llegar a la "universalidad terminológica" en el área de la morfología.

\section{Terminología: Enfoque multidisciplinario.}

Terminología como disciplina científica.Es conveniente señalar aquí que un término es una denominación lingüística de un concepto. Tanto en el campo de las ciencias morfológicas como en el resto de las ciencias, se recurre al discurso científico para comunicar los conocimientos de la disciplina. Para transmitir su mensaje, el discurso científico utiliza una lengua especializada, que debe ser la misma en toda la comunidad científica y debe extenderse por el mundo sin interferencias para su fácil y adecuada aplicación. La tarea de perfeccionar este código lingüístico específico para lograr su universalidad corresponde a la terminología (Bazán-Delgado, 2014).

La terminología es un campo de estudio concebido por Eugen Wüster en Viena, en la década de 1930, que la definió como un instrumento necesario para desambiguar la comunicación científica y técnica y llegar así a un entendimiento universal (Cabré, 2000).

Se la considera interdisciplinaria, ya que su estudio involucra otros campos de conocimiento: la lingüística y las ciencias del conocimiento, de la información y de la comunicación (Cabré; Mejías). 
La lingüística estudia la estructura de las lenguas, su evolución histórica y el conocimiento que los hablantes poseen de su propia lengua. Presta atención además al sistema cognitivo que hace posible que un hablante utilice el lenguaje para expresarse.

Las ciencias del conocimiento estudian las circunstancias históricas, sociológicas y psicológicas en las que surge el conocimiento y los criterios por los que se lo justifica o invalida.

Las ciencias de la información se encargan de recopilar, conservar, organizar, buscar y divulgar la información científica y tecnológica. Investigan además la estructura, las propiedades y los procesos de transmisión de la información y se vincula con los medios de procesamiento de la información.

Las ciencias de la comunicación estudian, analizan y discuten el sistema de la comunicación, es decir, el intercambio de información entre individuos. Se relacionan con la semiótica, que es la ciencia que estudia los sistemas de signos que permiten la comunicación.

Dificultades y desafío. A través de la terminología morfológica se estudia el vocabulario de este campo científico en particular y mediante la terminología morfológica internacional se brindan normas técnicas internacionales.

Aquellos profesores e investigadores morfólogos que entienden, organizan y estructuran la terminología morfológica internacional son verdaderos expertos o especialistas en su tema pero deben tener en cuenta los conocimientos necesarios para analizar el término desde otros puntos de vista: lingüístico, social y comunicacional.

Las unidades terminológicas -como se ha explicadoson a su vez unidades del lenguaje, elementos de cognición, vehículos de comunicación y elementos informáticos que deben almacenarse en bases de datos (Cabré).

Tal vez para ponerse de acuerdo con aquellos términos que presentan una mayor discusión en estos foros y que originan mayores problemas de aceptación por la comunidad médica, sea necesario abordarlos de una manera interdisciplinaria.

Este enfoque interdisciplinario enriquecería esta tarea terminológica para solucionar estos problemas. En síntesis, brindar un enfoque multidisciplinario y normativo para que sea usado por todos y establecer así la univocidad de los términos para evitar ambigüedades y problemas de comunicación.
Sistemas conceptual y terminológico. Una metodología es partir del concepto y no de la palabra. Primero se identifican y establecen los conceptos de la materia, que existen "a priori" en la mente de los profesionales, y luego se establecen sus correspondientes denominaciones. Para esto es necesario establecer el conjunto de características más comunes que representará ese concepto, dejando de lado los rasgos menos comunes aunque a veces más representativos desde el punto de vista cultural. En cuanto a la denominación de ese concepto, la normalización supone eliminar toda variedad denominativa para lograr un único término (Cabré).

Sin embargo, en el caso de los términos morfológicos el proceso generalmente ocurre al revés. La denominación en muchos casos ya existe en latín, por lo que no es necesario inventar un nombre sino buscar el equivalente más adecuado al término original y que a su vez refleje el concepto (Mejías Stuven; Vandaele \& Gingras Harvey).

Podría decirse entonces que hay una doble metodología de trabajo: una onomasiológica (del concepto a la palabra) y la otra semasiológica (de la palabra al concepto). Lo que queda claro en ambos casos es que los sistemas conceptual y terminológico son independientes entre sí.

El concepto de una estructura morfológica y su contexto son elementos de una realidad. Por esta razón, hay términos impregnados de regionalismos y no resulta sencillo desterrarlos. Los expertos buscan promover el buen uso terminológico y construir así una nueva realidad científica, cultural y social que perdure.

Esta tarea es compleja. El uso de los términos va más allá de las normas: el hecho de que se designe una denominación "oficial” y se eliminen las demás variedades no quiere decir que a partir de ese momento se va a respetar la norma en todos los ámbitos. En general el uso va por delante de la norma y es difícil revertir la situación.

\section{Lexicología, lexicografía y terminografía: disciplinas re-} lacionadas . Las tres disciplinas se relacionan con la lingüística. El sufijo -logía significa teoría y el sufijo -grafía, puesta en práctica. Las dos primeras trabajan sobre el léxico, es decir, sobre el conjunto de palabras que forman una lengua. La lexicología lo hace desde un punto de vista teórico, mientras que la lexicografía desde el práctico.

La lexicología estudia las palabras, su etimología (origen), su clasificación y las relaciones semánticas que se establecen entre ellas. Busca hacer generalizaciones sobre la estructura del léxico y el modo en el que la lengua representa ciertas áreas semánticas. 
La lexicografía es una ciencia instrumental que tiene como objetivo la elaboración y el análisis de los diccionarios. Estudia cada palabra de manera aislada y se centra en una lengua en particular. Su estudio es semasiológico.

La lexicografía tiene puntos en común con la terminografía, que se ocupa de la investigación y de la elaboración de diccionarios especializados, glosarios, bases de datos terminológicas, entre otros.

Conocer la existencia de estas tres disciplinas permite ampliar nuestros horizontes e incorporarlas a la tarea que realizan los expertos morfólogos a la hora de analizar y discutir la terminología anatómica, histológica y embriológica.

\section{DISCUSIÓN}

Un poco de historia: origen de la problemática. En el pasado no existían los medios de comunicación actuales, por lo que se ignoraba lo que se escribía en otros países. Esto originó la proliferación de diferentes términos para una misma estructura.

Por otra parte, las guerras, el ultra-nacionalismo, el poder militar y la prepotencia hegemónica de turno propiciaron la persistencia de algunos nombres en lugar de otros. A esto se le sumaban las discrepancias científicas entre los diferentes anatomistas (Duque Parra et al., 2006; Whitworth, 2007; Woywodt \& Matteson, 2007; Cruz et al., 2010a).

A fines del siglo XIX, existían alrededor de 50.000 nombres anatómicos para 5.000 estructuras. Ello propiciaba la confusión y el caos en la terminología, lo cual a su vez dificultaba el intercambio de trabajos científicos, especialmente los anatómicos. Por esta razón anatomistas de diferentes partes del mundo decidieron reunirse con el objeto de unificar los criterios y determinar un idioma universal en las ciencias anatómicas.

El primer comité de expertos fue fundado por la Sociedad Alemana de Anatomía en 1895 y señaló más de 5.000 términos anatómicos con un solo nombre, reunidos en un libro denominado Nomina Anatomica de Basilea (BNA). En 1955, en París, se corrigió y aumentó, reuniéndose en un nuevo libro: Nomina Anatomica de Paris (PNA), tal vez la de mayor repercusión internacional de todas las nomenclaturas o Nomina, ya que fue publicada con la ayuda financiera de la UNESCO. A esta última le sucedieron otras hasta que en 1989, en Brasil, se estableció el Comité Internacional de Terminología Anatómica (FCAT) (Duque Parra et al.,
2002; Álvarez \& Campohermoso, 2007; Cruz et al., 2010a; Algieri et al., 2011; Vandaele \& Gringas Harvey). Así se reemplazó la denominación de Nomina por Terminologia (Whitmore, 2009).

En Latinoamérica, hubo esfuerzos individuales de eximios anatomistas, como en Argentina y Colombia, quienes propusieron un listado de términos anatómicos en castellano (Provenzano, 1951; Chatain, 1967) y que deberían ser comparados con los de la publicación de la terminología española (Cruz et al., 2010a). También se ha observado que existe un progresivo interés de los morfólogos y de áreas afines a las ciencias médicas y biológicas en publicar sus artículos utilizando la terminología internacional, lo que muestra un mayor compromiso en este tema (del Sol, 2001).

Problemática actual. Existen problemas con algunos términos imprecisos que se utilizan en la práctica quirúrgica profesional y también con las numerosas denominaciones que se le atribuyen a una misma estructura en publicaciones médicas actuales (Grinberg, 1999; Duque Parra et al., 2002; Jiménez-Gutiérrez; Vogl, 2009; Matusz, 2010; Cantín \& Suazo Galdames, 2011; Vandaele \& Gingras Harvey). Asimismo las denominaciones vulgares se ven incrementadas en el ámbito de la ciencia popular, en los manuscritos e incluso en textos no médicos (Bezerra et al., 1993; Bezerra et al., 1999).

Por otro lado, los términos inadecuados llevan a una visión alterada de la realidad morfológica y desorientan a estudiantes, graduados, profesores y autores (Cruz et al., 2010a; Duque Parra et al., 2012; Bazán-Delgado, 2014; Vásquez \& del Sol, 2014).

La dimensión comunicativa de estos términos morfológicos es muy amplia, no sólo en cuanto a su geografía sino también a las diversas especialidades y profesionales de la salud que existen. Esto es lo que dificulta que un término sea fácilmente universal.

Hoy en día el conocimiento ha avanzado y se cuenta con herramientas que pueden ayudar a resolver este problema desde otra perspectiva. Entre ellas, la terminología como disciplina surgió y se desarrolló.

Últimamente algunos morfólogos señalan en sus trabajos de terminología anatómica, histológica y embriológica la relación con la lingüística (Duque Parra et al., 2006; Salgado \& Trujillo; Mejías Stuven). Por otro lado, algunos lingüistas indican en sus trabajos de lingüística la relación con la terminología morfológica (Jiménez-Gutiérrez; Vandaele \& Gingras Harvey). Estas observaciones aisladas demuestran las carencias que ambos grupos de profesiona- 
les tienen para tratar este tema. Tal vez sea el momento de compartir el diálogo y la discusión de estos temas en foros interdisciplinarios.

Por otra parte, todos ellos en nuestra región IberoLatinoamericana realizan un esfuerzo para mejorar el uso apropiado del lenguaje, tanto del español como del portugués, evitando el deterioro del idioma.

En el estatuto del SILAT, en el capítulo dos, titulado "Objetivos y finalidades" (artículo dos, inciso dos) dice: "Estudiar y analizar la traducción en español y portugués de la terminología morfológica internacional" (Losardo et al., 2010). Creemos que esta tarea es interdisciplinaria y permitirá estrechar vínculos y compartir conocimientos entre los profesionales morfólogos y lingüistas.

Entonces, resultaría imprudente desaprovechar el conocimiento que brinda esta disciplina que seguramente permitirá resolver algunos de los obstáculos que hoy día siguen existiendo.

\section{CONCLUSIÓN}

Si bien hace más de cien años que se está tratando de lograr la utilización de una terminología morfológica unificada en todos los ámbitos académicos del mundo, el problema sigue sin resolverse por completo, lo que demuestra que es una tarea compleja.

En la región Ibero-Latinoamericana, en los últimos tiempos y gracias al SILAT, creció el interés y se logró un mayor avance. Estos profesores e investigadores que conforman este grupo de expertos lo han podido transmitir a sus colegas, logrando una progresiva toma de conciencia acerca de su pertenencia y compromiso a sus lenguas. De esta manera han brindado una valiosa ayuda en el proyecto internacional en pos de una mejor calidad de educación y de ciencia.

Sin embargo, el enfoque que se utiliza es el mismo desde ese primer encuentro en Basilea a fines del siglo XIX y sigue existiendo un número de términos que aún no se resuelven. El concepto y la denominación de una estructura morfológica y su contexto son elementos de una realidad difícil de modificar.

El uso de los términos va más allá de las normas que un grupo de expertos quiera imponer al resto de la comunidad científica. Designar una denominación "oficial" y eliminar las demás variedades no significa que a partir de ese momento se adopte dicha norma, ya que el uso va siempre por delante de la norma y es difícil revertir la situación a corto plazo.

La terminología como disciplina se inició hace más de setenta años y su estudio trae consigo otras ramas del saber, como la lingüística, la comunicación, el conocimiento y la informática. Sin embargo, todavía no ha tenido un punto de encuentro con el esfuerzo que realizan los morfólogos en esta tarea de unificar la terminologías anatómica, histológica y embriológica.

Bajo esta perspectiva el término no sólo es una palabra que hace referencia a una estructura morfológica propia de la medicina, sino que también es una unidad del lenguaje, un vehículo de comunicación, un elemento cognitivo e informático.

Quizás sea el momento de probar un enfoque distinto y recopilar aquellos términos difíciles que desde el método tradicional no se han podido resolver y analizarlos desde un punto de vista lingüístico, social y comunicacional, con el fin de promover el buen uso terminológico y construir así una nueva realidad científica, cultural y social que perdure.

En síntesis, resulta necesario un conocimiento adicional de estas disciplinas para que el estudio de los expertos morfólogos sea más efectivo.

\section{AGRADECIMIENTOS}

A la señorita Bárbara Sol Moschini del Instituto de Enseñanza Superior en Lenguas Vivas "Juan Ramón Fernández" (Buenos Aires) por sus aportes en las búsquedas y traducciones bibliográficas así como en la redacción final del trabajo.

LOSARDO, R. J.; PRATES, N. E. V. B.; ARTEAGAMARTÍNEZ, M.; CABRAL, R. H. \& GARCÍA-PELÁEZ, M. I. International Morphological Terminology: more than anatomy, histology and embryology. Int. J. Morphol., 33(1):400-407, 2015.

SUMMARY: The study of the International Morphological Terminology and its implementation in a system of rules is complex. The work done by the group of experts in morphology is not enough. Some aspects that will get us closer to the understanding of this problem will be explained and a way of solving it will be shown.

KEY WORDS: Terminology; Anatomy; Histology; Embryology; Morphology; Medical education; Linguistics. 


\section{REFERENCIAS BIBLIOGRÁFICAS}

Algieri, R. D.; Ferrante, M. S. \& Pró, E. Analysis of the General Surgeon's knowledge of International Anatomical Terminology (IAT). Int. J. Morphol., 31(4):1511-6, 2013.

Algieri, R. D.; Pró, E. A.; Forlizzi, V. \& Ferrante, M. S. Reseña de la evolución histórica de los términos anatómicos. Rev. Arg. Anat. Online, 2(4):106-11, 2011.

Álvarez, G. O. \& Campohermoso, R. O. Evolución histórica conceptual de la Terminología Anatómica. Rev. Cuad., 52(1):1137, 2007.

Andriani, O. C. La torre de Babel, París y Brisbane: un recorrido sobre sus influencias en la terminología de la segmentación hepática. Rev. Arg. Anat. Online, 1(3):84-8, 2010.

Bazán-Delgado, A. La biología y su lenguaje científico, un análisis lingüístico. Santiago, 133:261-71, 2014.

Bezerra, A. J. C.; Bezerra, A. S. A. \& Didio, L. J. A. Yanomani Indians and the anatomical and biomedical terms in their language. Rev. Chil. Anat., 11(1):25-33, 1993.

Bezerra, A. J. C.; Didio L. J. A. \& Piva-Júnior, L. Términos anatómicos en la Ilíada de Homero. Rev. Chil. Anat., 9(1):73-7, 1999.

Cabré, M. T. Terminologie et linguistique: la théorie des portes. Terminol. Nouv., 21:10-15, 2000.

Cantín, M. \& Suazo Galdames, I. Cleft lip in biomedical terminology. Rev. Med. Chil., 139(2):274-5, 2011.

Cruz Gutiérrez, R.; Rodríguez Torres, A.; Prates, J. C.; Losardo, R. J. \& Valverde Barbato, N. Ibero-Latin-American Symposia Terminology. Anatomy, Histology and Embryology. Int. J. Morphol., 28(1):333-6, 2010a.

Cruz Gutiérrez, R.; Rodríguez Torres, A.; Prates, J. C.; Losardo, R. J. \& Valverde Barbato de Prates, N. Simposio Ibero-latinoamericano de Terminología Morfológica. Características generales. Int. J. Morphol., 28(2):643-6, 2010b.

Chatain, I. Terminología anatómica. Cali, Norma, 1967.

del Sol Calderón, M. Las ciencias morfológicas y su difusión a través de la Revista Chilena de Anatomía. Rev. Chil. Anat., 19(2):129-30, 2001.

del Sol, M. \& Vásquez, B. Median cephalic and median basilic veins. Why their exclusion from Terminologia Anatomica? Int. J. Morphol., 32(2):721-4, 2014.

Duque Parra, J. E.; Barco Ríos, J. \& Duque Quintero, N. Refining the Anatomical Terminology: Opening and Foramen, Two Similarities and Redundancy. Int. J. Morphol., 30(4):14979, 2012.

Duque Parra, J. E.; Gómez Arias, N. C. \& Giraldo R., D. P. Nomenclatura Anatómica Internacional. Medicina U. P. B., 21(1):43-55, 2002.

Duque-Parra, J. E.; Llano-Idárraga, J. O. \& Duque-Parra, C. A. Reflections on eponyms in neuroscience terminology. Anat. Rec. B New Anat., 289(6):219-24, 2006.

Duque, J. E. \& Ríos, J. Umbilical-spinous line: a morphological term that should be included in the anatomical terminology. Colomb. Med. (Cali), 44(3):202-4, 2013.

Federative Committee on Anatomical Terminology (FCAT). Terminología Anatómica: International Anatomical Terminology. Stuttgart, Georg Thieme Verlag, 1998.

Federative International Committee on Anatomical Terminology (FICAT). Terminologia Histologica: International Terms for Human Cytology and Histology. Philadelphia, Wolters Kluwer/Lippincott Williams \& Wilkins, 2008.

Federative International Programme on Anatomical Terminology (FIPAT). Terminologia Anatomica: International A

Grinberg, M. Epônimos em Cardiologia. Homenagem e exemplo. São Paulo, Livraria Roca, 1999.

International Federation of Associations of Anatomists. Rev. Chil. Anat., 10(2):139-44, 1992.

Jiménez-Gutiérrez, I. La sinonimia y la polisemia en la terminología anatómica: términos de ubicación y de relación de estructuras anatómicas. Entrecult., 1:579-97, 2009.

Losardo, R. J. Pan-American Association of Anatomy: Historic Review and Eminent Rules. Int. J. Morphol., 27(4):1345$52,2009$.

Losardo, R. J.; Cruz Gutiérrez, R.; Rodríguez Torres, A.; Prates, J. C. \& Valverde Barbato de Prates, N. Iberia-Latin-American Symposia of Morphological Terminology (SILAT). First Two Years and Statute. Int. J. Morphol., 28(4):1323-6, 2010.

Matusz, P. Misleading Latin/English equivalents for some liver terms in Terminologia Anatomica. Anat. Sci. Educ., 3(3):1567, 2010.

Mejías S. R. El latín como lengua de la terminología anatómica: algunas observaciones sobre su rol. Rev. Arg. Anat. Online, 3(2):33-42, 2012. 
LOSARDO, R. J.; PRATES, N. E. V. B.; ARTEAGA-MARTínEZ, M.; CABRAL, R. H. \& GARCÍA-PELÁEZ, M. I. Terminología morfológica internacional: algo más que anatomía, histología y embriología. Int. J. Morphol., 33(I):400-407, 2015.

Pawlina, W. \& Drake, R. Moving forward with Terminologia Anatomica. Anat. Sci. Educ., 2(3):93, 2009.

Provenzano, S. D. Nómina anatómica. Buenos Aires, El Ateneo, 1951.

Salgado, A. G. E. \& Trujillo, H. E. Terminología: Historia e importancia en el desarrollo de las ciencias morfológicas. Forensic Oral Pathol. J., 1(1):9-11, 2010.

Sociedad Anatómica Española (SAE). Terminología Anatómica. Madrid, Médica Panamericana, 2001.

Vandaele, S. \& Gingras Harvey, M. Les nomenclatures anatomiques: histoire et traduction. Panacea, 14(38):222-34, 2013.

Vásquez, B. \& del Sol, M. The Terminologia Histologica in the Medical Sciences. Int. J. Morphol., 32(1):375-80, 2014.

Vogl, A. W. Awareness of and access to a unified terminology by anatomists. Anat. Sci. Educ., 2(3):139-40, 2009.

Whitmore, I. Terminologia Anatomica includes terms in English for all scientists writing in English. Anat. Sci. Educ., 2(3):141, 2009.

Whitworth, J. A. Should eponyms be abandoned? No. BMJ, 335(7617):425, 2007.

Woywodt, A. \& Matteson, E. Should eponyms be abandoned? Yes. BMJ, 335(7617):424, 2007.
Dirección para Correspondencia

Prof. Dr. Ricardo Jorge Losardo

Lavalle 1844 - Piso $5^{\circ}$ - Oficina 30 (1051)

Ciudad de Buenos Aires

ARGENTINA

Email: ricardo.losardo@salvador.edu.ar

Recibido : 27-10-2014

Aceptado: 27-11-2014 